\title{
COMPARATIVE EVALUATION OF NARROW BAND UVB VERSUS ORAL 8-METHOXYPSORALEN UVA IN CHRONIC PLAQUE PSORIASIS
}

\author{
Rachita Talwar', Ranjeeta Sapam², Arpana Rijal ${ }^{3}$ \\ ${ }^{1}$ Specialist, Department of Dermatology, NMC Speciality Hospital. \\ ${ }^{2}$ Associate Professor, Department of Dermatology, JNIMS, Imphal. \\ ${ }^{3}$ Professor, Department of Dermatology, BPKIHS, Nepal.
}

\section{ABSTRACT}

\section{BACKGROUND}

Psoriasis is a chronic, disfiguring, inflammatory and proliferative condition of the skin.

The aim of this study was to study the effect of narrowband UVB (NB-UVB) and psoralen ultraviolet A (PUVA) in the treatment of chronic plaque psoriasis, and to compare the therapeutic efficacy of both.

\section{MATERIALS AND METHODS}

This was a randomised, intraindividually controlled paired comparison study. Each side of the patient's upper extremity was treated independently, with NB-UVB and PUVA. PUVA was given in a dose of 70\% of MPD (minimal phototoxic dose) or depending upon the skin type and NB-UVB were given in a dose of $70 \%$ of MED (minimal erythema dose). An increment of $20 \%$ was made at each session till minimally perceptible erythema occurred. The assessment was done by calculating the MPASI un score at each visit up to $75 \%$ improvement.

\section{RESULTS}

Thirty patients were enrolled out of which 3 defaulted. The number of patients achieving $75 \%$ improvement in the MPASI UL $_{\text {were }} 23$ on the PUVA side and 22 on the NB-UVB side, the number of treatments required was 15.43 on PUVA side 16.09 on the NB-UVB side and the time taken was 34.26 and 36.86 days for PUVA and NB-UVB sides respectively. The adverse effects were less with NB-UVB.

\section{CONCLUSION}

There was no significant difference between the two modalities in the number of patients achieving the number of treatments

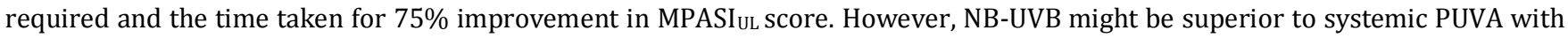
better tolerance and low profile of side effects in the treatment of psoriasis.

\section{KEYWORDS}

NBUVB, PUVA, Psoriasis, Randomisation, Trial.

HOW TO CITE THIS ARTICLE: Talwar R, Sapam R, Rijal A. Comparative evaluation of narrowband UVB verses oral 8methoxypsoralen UVA in chronic plaque psoriasis. J. Evolution Med. Dent. Sci. 2016;5(97):7111-7115, DOI: 10.14260/Jemds/2016/1610

\section{BACKGROUND}

Psoriasis is a common, chronic, disfiguring, inflammatory and proliferative condition of the skin, in which both genetic and environmental influences have a critical role. ${ }^{1}$

Photochemotherapy using psoralen has been a time tested treatment for psoriasis. A significant advance in the phototherapy has been the introduction of narrowband UVB (NB-UVB) therapy using Phillips model TL 01 lights having spectrum of $310-315 \mathrm{~nm}$ with a peak at $311 \mathrm{~nm}$. NB UVB has been found to be as effective as PUVA $^{2}$ without distinct disadvantages of the latter and considered superior to broad band $\mathrm{UVB}^{3}$ and bath PUVA.4,5 Various studies 2,6 have been carried out in the western countries comparing the efficacy of NB-UVB and PUVA phototherapy with contradictory results. The present study is undertaken to compare the therapeutic efficacy of narrow band (TL01) UVB phototherapy (NB-UVB)

Financial or Other, Competing Interest: None.

Submission 28-10-2016, Peer Review 21-11-2016,

Acceptance 28-11-2016, Published 05-12-2016.

Corresponding Author:

Ranjeeta Sapam,

Moirangkhom, Sougaijam Leirak,

Imphal.

E-mail:ranssapam@gmail.com

DOI: $10.14260 /$ jemds $/ 2016 / 1610$ with oral 8-methoxypsoralen photochemotherapy (PUVA) in patients with chronic plaque psoriasis.

\section{MATERIALS AND METHODS}

All clinically diagnosed cases of chronic plaque psoriasis with involvement of $>20 \%$ of body surface area (using rule of nine) and bilaterally symmetrical involvement in the upper limbs attending the Dermatology Out Patient Department of BPKIHS, Nepal from April 2006 to April 2007 were included in the study. Exclusion criteria included age less than 14 years or $>70$ years, pregnant and lactating mothers, renal, hepatic or cardiovascular disease, who had taken any antipsoriatic treatment within the last 4 weeks, received any form of UV therapy within the preceding 6 months, any radiation therapy or previous failure/intolerance to phototherapy, or on any photosensitive drugs and with cataract or skin cancer. Prior informed and written consent was taken from all patients. A detailed history and complete clinical examination was done. A wash off period of 4 weeks was given to patients on any kind of treatments. No concomitant therapy was allowed except for emollients and antihistamines.

Baseline investigations of routine blood counts, liver function tests and renal function tests were performed and repeated at 3 weekly intervals. 
The present study was designed to do half side comparison in the same patient between the two modalities. Upper extremity was chosen as the site considering the ease with which one side of the upper limb could be shielded and also to reduce the inconvenience caused to the patient.

In the present study, assessment was done by calculating the psoriasis area severity index (PASI) score $^{7}$ for only the upper limbs, separately for each side at baseline. Therefore, the PASI score was modified for the upper limbs (MPASIuL). Ralloc software was used for randomisation of the allocation of side.

Determination of MED and MPD ${ }^{8}$-- Prior to the study, first the minimal erythema dose (MED) of NB-UVB was determined in each patient using test doses 100,140, 200, 280, 390, 550, 770, $1100 \mathrm{~mJ} / \mathrm{cm}^{2}$ with the help of a photo testing template. The first 6 doses were used for skin type I and II and the last 6 for types III through VI. MED was defined as the smallest dose of radiation required to achieve just detectable erythema, read after 24 hours. Subsequently, the minimal phototoxic dose (MPD) of PUVA was determined in each patient using test doses $0.5,1,1.5,2.0,3.0,5.0,7.0,9 \mathrm{~J} / \mathrm{cm}^{2}$. The first 6 doses were used for skin type I and II and the last 6 for types III through VI. MPD was defined as the smallest dose of radiation required to achieve just detectable erythema and was read after 72 hours.

\section{Phototherapy}

On the first treatment day, patient received NB-UVB irradiation on one side of the extremity. Such NB-UVB sessions were planned 3 times weekly every alternate day. ${ }^{2}$ During the irradiation, the rest of the body was covered by tightly woven cloth to prevent the transmission of UV light. On the second day, the patient was given oral 8-methoxypsoralen capsules in a dose of $0.6 \mathrm{mg} / \mathrm{kg}$ body weight. 9 Two hours later, the other side of the patient's extremity and the remaining part of body was exposed to UVA while the body area previously treated with NBUVB was shielded from receiving additional UVA radiation. These PUVA sessions were given 3 times weekly ${ }^{9}$ alternating with the NB-UVB sessions.

The initial irradiation dose given for NB-UVB was $70 \%$ of MED and for PUVA it was 70\% of MPD. 8 Patients with a negative MED were treated with a standard starting dose ${ }^{9}$ of $280 \mathrm{~mJ} / \mathrm{cm}^{2}$ (70\% of MED for skin type IV, V) for NB-UVB. Patients with a negative MPD were treated with skin type based initial UVA dose of $1.5 \mathrm{~J} / \mathrm{cm}^{2}$ for skin type III, $2 \mathrm{~J} / \mathrm{cm}^{2}$ for skin type IV and $2.5 \mathrm{~J} / \mathrm{cm}^{2}$ for skin type V.10,11 Subsequent dose increments were aimed at eliciting or maintaining a slight erythema. The irradiation dose was increased by $20 \%$ of the previous dose if there was an absence of erythematous response. If there was Grade 1 erythema, same dose as previous visit, thereafter reduce the increments and if Grade 2 erythema, next exposure is postponed until erythema resolves; and then repeat the previous dose: subsequently, reduce increments. The half side treatment was performed until there was $\geq 75 \%$ improvement in the MPASIuL score or for a maximum period of 30 sessions whichever was earlier.
Light therapy was administered using "Derma India, Chennai Lightning" cubicles. During phototherapy, all patients wore UV protective goggles and the genitalia were shielded.

All the patients were examined by the dermatologist not aware of the treatment allocation. The assessment was done at each visit up to $75 \%$ improvement in the MPASI uL or up to 30 sessions whichever was earlier.

\section{According to the Response the Patients were Classified as those Having}

- Marked Improvement: $\geq 75 \%$ improvement in MPASI ${ }_{U L}$ score from the baseline within a maximum of 30 sessions.

- Partial Improvement: $1 \%-74 \%$ improvement in MPASI score from the baseline within a maximum of 30 sessions.

- Static Disease: No improvement in MPASIuL scores.

- Disease Worsening: Increase in MPASIul score.

Any adverse effect during the treatment period was recorded. Clinical photographs were taken. Outcome measures included improvement in the MPASIuL scores, number of exposures for the improvement, cumulative UV dose for the improvement and any adverse effect within that period.

\section{Statistical Analysis}

Data were entered in Microsoft Excel 2000 and converted into SPSS version 10.0 for statistical analysis. For categorical and continuous data, simple percentage, mean and standard deviation were calculated. To find out the significant difference among the groups Chi Square was calculated for categorical data and Independent $t$ test was calculated for numerical data. Significance was defined as $\mathrm{p}<0.05$.

\section{RESULTS}

Of the 30 patients, 27 completed the study. Out of 27 patients, there were $16(59.3 \%)$ males and $11(40.7 \%)$ females and male to female ratio was $1.45: 1$. The age of the patients ranged from $14-70$ years with the mean age of $35.7 \pm 14.82$ years. The body surface area (BSA) involvement varied among patients. Five $(18.5 \%)$ patients had up to $25 \%$ BSA involvement, 6 (22.2\%) had 26-50\% involvement, 10 (37.03\%) had 51-75\% involvement and 6 (22.2\%) had 76-100\% involvement.

\section{MPASI $_{\text {uL }}$ Scores}

The baseline psoriasis area and severity index (MPASI $\left.\mathrm{UL}_{\mathrm{L}}\right)$ ranged from 18 to 72 with the mean being $44.04 \pm 15.46$ for both the groups. The final MPASIuL for PUVA side was $13.00 \pm$ 9.12 (range, 4-48) and for NB-UVB side was $13.67 \pm 10.10$ (range, 4-48). There was no statistical difference in these parameters between the two sides ( $\mathrm{p}>0.05)$.

There was a statistically significant improvement in MPASI UL score with both the modalities, PUVA and NB-UVB.

\section{Outcome of Treatment}

The outcome is depicted in table no 1 . There was no statistically significant difference in these parameters of response between the two sides ( $\mathrm{p}>0.05)$. 


\begin{tabular}{|c|c|c|c|}
\hline & $\begin{array}{c}\text { PUVA side } \\
(n=27)\end{array}$ & $\begin{array}{c}\text { NB-UVB side } \\
(n=27)\end{array}$ & p value \\
\hline No. of patients with marked improvement ( $\downarrow$ MPASIUL $\geq 75 \%)$ & $23(85.18 \%)$ & $22(81.48 \%)$ & 0.718 \\
\hline No. of patients with partial response (\MPASI $\left.{ }_{\text {UL }} 1 \%-74 \%\right)$ & $3(11.11 \%)$ & $4(14.81 \%)$ & 0.500 \\
\hline No. of patients with disease worsening & $1(3.7 \%)$ & $1(3.7 \%)$ & 0.754 \\
\hline $\begin{array}{l}\text { No. of treatments for marked improvement (Mean } \pm \text { SD) } \\
\text { Range }\end{array}$ & $\begin{array}{l}15.43 \pm 2.46 \\
12-21\end{array}$ & $\begin{array}{c}16.09 \pm 2.29 \\
12-22\end{array}$ & 0.36 \\
\hline $\begin{array}{l}\text { Time taken for marked improvement in days (Mean } \pm \text { SD) } \\
\text { Range }\end{array}$ & $\begin{array}{c}34.26 \pm 5.67 \\
26-47 \\
\end{array}$ & $\begin{array}{c}36.86 \pm 5.43 \\
27-51 \\
\end{array}$ & 0.123 \\
\hline $\begin{array}{l}\text { Total UV dose required for marked improvement in } \mathrm{J} / \mathrm{cm}^{2} \\
\text { (Mean } \pm \text { SD) } \\
\text { Range }\left(\mathrm{J} / \mathrm{cm}^{2}\right)\end{array}$ & $\begin{array}{l}127.39 \pm 36.97 \\
75.87-210.87\end{array}$ & $\begin{array}{l}33.47 \pm 16.81 \\
11-75.3\end{array}$ & 0 \\
\hline
\end{tabular}

Cumulative number of patients who achieved marked improvement is depicted in table no 2 .

\begin{tabular}{|c|c|c|c|}
\hline $\begin{array}{c}\text { Number of } \\
\text { Sessions }\end{array}$ & $\begin{array}{c}\text { PUVA side } \\
(\mathbf{n = 2 7 )}\end{array}$ & $\begin{array}{c}\text { NB-UVB } \\
\text { side } \\
(\mathbf{n = 2 7})\end{array}$ & $\begin{array}{c}\mathbf{p} \\
\text { value }\end{array}$ \\
\hline 12 & $2(7.40 \%)$ & $1(3.70 \%)$ & 0.5 \\
\hline 14 & $10(37.03 \%)$ & $5(18.51 \%)$ & 0.22 \\
\hline 16 & $16(59.25 \%)$ & $14(51.85 \%)$ & 0.58 \\
\hline 18 & $21(77.77 \%)$ & $19(70.37 \%)$ & 0.53 \\
\hline 20 & $22(81.48 \%)$ & $21(77.77 \%)$ & 0.73 \\
\hline 22 & $23(85.18 \%)$ & $22(81.48 \%)$ & 0.5 \\
\hline \multicolumn{4}{|c|}{ Table 2. Cumulative Number of Patients who Achieved } \\
\hline
\end{tabular}

\section{Changes in MPASIuL Score on treatment}

After 2 sessions the mean MPASIuL in the PUVA side was $43.33 \pm 15.26$ and in the NB-UVB side was $43.89 \pm 15.52$. After 4 sessions it was $39.52 \pm 315.05$ in PUVA side and $41.81 \pm 14.86$ in NB-UVB side. At the end of 16 sessions, mean MPASI UL was $18.71 \pm 11.76$ in PUVA side and $18.32 \pm 12.56$ in NB-UVB side. There was no statistically significant difference in the mean MPASI $_{\text {uL }}$ between the two sides till the end of 16 sessions (Fig. 1).

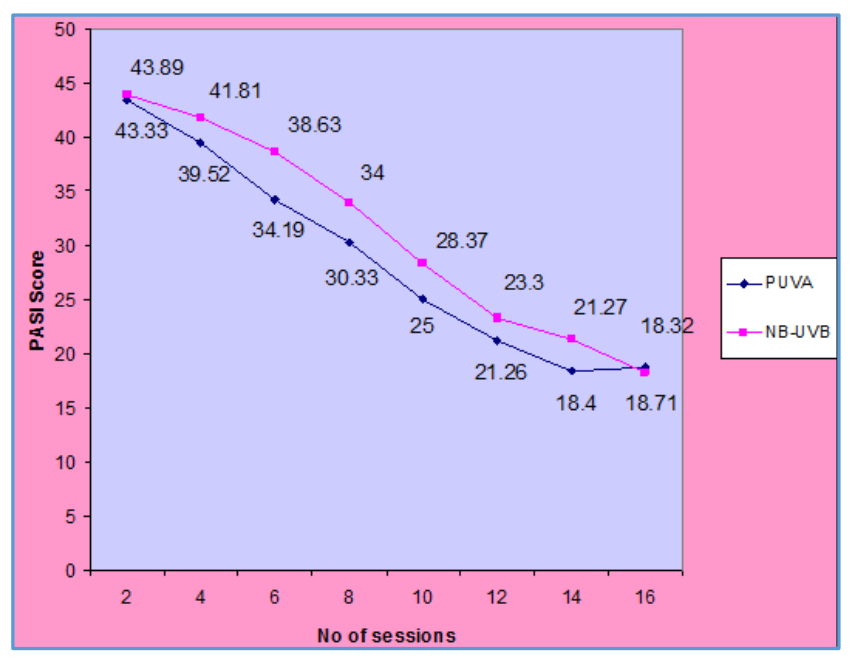

Figure 1. Changes in MPASI ${ }_{U L}$ on Treatment

\section{Adverse Effects}

Eight (29.6\%) patients in PUVA side and 7 (25.9\%) patients in NB-UVB side experienced side effects. Two (7.4\%) patients in each group developed polymorphic light eruption (PMLE). Exacerbation of pruritus was experienced by 6 (22.2\%) patients on PUVA side and $5(18.5 \%)$ patients on NB-UVB side, which was managed with antihistamines and emollients. Six (22.2\%) patients who experienced pruritus on PUVA side also had nausea due to intake of oral Methoxypsoralen tablet; it was managed with oral antiemetics half an hour before methoxypsoralen dose.

\section{DISCUSSION}

Although different modalities of treatment can induce remission in a high percentage of psoriatic patients, in the absence of a cure, both safe and effective therapy to maintain patients in prolonged remission is not found.

Different studies 2,6 have compared the efficacy of PUVA and NBUVB with conflicting reports as to which is superior.

Since the present study was a within-patient side-to-side comparison study, one half of the upper extremity served as a control for the other half. Similar kind of side-to-side comparison studies have been done by van Weelden et $\mathrm{al}^{12}$ and Tanew et $\mathrm{al}^{2}$ and in the past for the comparison of PUVA and NB-UVB and by Dawe et $\mathrm{al}^{4}$ for comparing bath PUVA and NBUVB. This kind of a study design is advantageous as it obviates the enrolment of controls.

The regimen used in the present study for both PUVA and NB-UVB was the thrice weekly regimen. Thrice weekly frequency chosen for PUVA was in accordance to the American PUVA protocol. For NB-UVB three times weekly appeared to be the optimal regimen for efficacy in that two NB-UVB sessions took $50 \%$ longer to clear psoriasis compared to three times weekly, 88 vs. 58 days respectively. ${ }^{13}$ Regimens similar to ours were used by Tanew et $\mathrm{al}^{2}$ and Tahir et al ${ }^{14}$ while van Weelden et $\mathrm{al}^{12}$ Gordon et $\mathrm{al}^{6}$ and Yones et al ${ }^{8}$ used the twice weekly regimens. A very different twice weekly frequency for PUVA and thrice weekly frequency for NB-UVB was used by Dawe et $\mathrm{al}^{4}$ and Markham et $\mathrm{al}^{15}$ in their studies. However, the kind of regimens used did not have any effect on the outcome of the study.

A 75\% reduction in PASI score called PASI 75 is the current benchmark of primary end point for most clinical trials. ${ }^{13}$ In the present study, there was no statistically significant difference in the number of patients who achieved reduction in MPASI $\geq 75 \%$ with both modalities $(85.18 \%$ on PUVA side and $81.48 \%$ on NB-UVB side; $p$ value 0.718 ). This is in agreement with the previous studies done by Tanew et $\mathrm{al}^{2}$ who also found statistically similar clearance rates of $43 \%$ for PUVA and $33 \%$ for NB-UVB, and Markham et al ${ }^{15}$ who found very high clearance rates of $100 \%$ for both PUVA and NB-UVB. Such high clearance rates can be explained on the basis that the patients in Markham's study were treated until they were completely clear. However, Dawe et $\mathrm{al}^{4}$ found significantly higher clearance rates with NB-UVB as compared to PUVA 
( $54 \%$ vs. $75 \%, p=0.03$ ). In contrast, in Gordon et al's ${ }^{6}$ study, clearance of psoriasis was achieved in a significantly greater proportion of patients treated with PUVA (84\%) than with NBUVB (63\%, $p=0.001)$. Similar results were found by Tahir et al14 who found the clearance rates of PUVA to be $85 \%$ as compared to $60 \%$ for NB-UVB $(\mathrm{p}=0.04)$ and by Yones et al 8 who found the clearance rates of PUVA and NB-UVB to be $84 \%$ and $65 \%$ respectively.

The number of treatments required to achieve $75 \%$ improvement with both modalities was analysed in the present study and it was found that there was no significant difference $(p=0.36)$. Similar results were obtained by Tanew et $\mathrm{al}^{2}$ who found that for $>75 \%$ reduction of PASI score using PUVA and NB-UVB, equal numbers of exposures i.e. 15 exposures are required. This differed from study by Markham et al 15 , who reported significantly fewer treatments with PUVA for marked improvement as compared to NB-UVB (19 vs 25.5, $\mathrm{p}=0.03$ ), although there was no significant difference in the number of days to clear. This may be due to twice weekly PUVA exposure as compared to thrice weekly regimen for NB-UVB in their study. Similarly other studies $4,6,8,14$ done by various authors have shown the median number of treatments to clearance, to be significantly lower for PUVA as compared to NB-UVB. The number of treatments to clear as reported by Gordon et al 6 was 16.7 for PUVA vs. 25.3 for NB-UVB ( $p<0.0001$ ), Dawe et $\mathrm{al}^{4}$ reported it to be 19 for PUVA and 24.5 for NBUVB ( $p=0.01$ ), Tahir et al ${ }^{14}$ found it to be as 17 for PUVA and 25.5 for NBUVB $(\mathrm{p}<0.001)$ and Yones et al ${ }^{8}$ similarly found it to be 17 for PUVA and 28.5 for NBUVB ( $p<.001$ ).

There was no statistically significant difference in the present study between the time taken to achieve $75 \%$ improvement in both sides $(\mathrm{p}=0.123)$. Tanew et al's ${ }^{2}$ study also concluded that statistically similar treatment duration of 38 days is required for $\geq 75 \%$ reduction of PASI scores using PUVA and NB-UVB. Markham et al ${ }^{15}$ similarly found no significant difference in the number of days to clear with both modalities $(p=0.46)$. In contrast, Dawe et $\mathrm{al}^{4}$ found that NBUVB achieved clearance in a median of 11 days ( $p=.001)$ more quickly than PUVA.

The mean cumulative PUVA dose for $75 \%$ improvement was $127.39 \pm 36.97 \mathrm{~J} / \mathrm{cm}^{2}$ and the mean NB-UVB dose was $33.47 \pm 16.81 \mathrm{~J} / \mathrm{cm}^{2}$ in this study. Yones et al ${ }^{8}$ reported that mean PUVA dose for $>75 \%$ improvement was $126 \mathrm{~J} / \mathrm{cm}^{2}$ and mean NB-UVB dose was $41.3 \mathrm{~J} / \mathrm{cm}^{2}$.

Since only the upper extremity was chosen as the area of evaluation in the present study, the anatomical area wise comparison of effectiveness of the two modalities could not be analysed as shown by van Weelden et $\mathrm{al}^{12}$, who concluded that PUVA was more effective in clearing extremities and NB-UVB was more effective in clearing trunk. His findings indicated that PUVA may be better for clearing lesions that are more recalcitrant to therapy, such as those found on the extremities. None of the patients in the present study came for followup after the $75 \%$ clearance, so the mean remission period with PUVA and NB-UVB could not be compared. Gordon et al ${ }^{6}$ had reported three times more remission period for PUVA as compared with NB-UVB $(12 \%$ of those treated with NBUVB were clear of psoriasis 6 months after finishing treatment compared with $35 \%$ for PUVA, $p=0.002$ ). Similarly Yones et al ${ }^{8}$ reported that 6 months after cessation of therapy, $68 \%$ of PUVA treated patients were still in remission vs. 35\% of NBUVB treated patients ( $p=.02)$. However, in Markham et al's15 study, an average remission period of 288.5 days for NBUVB and 231 days for PUVA group, was observed during the followup of 1 year, which was not found to be statistically significant.

In the present study, $1(3.7 \%)$ patient had a flare up of disease in the form of grade 2 erythema and an increased scaling which was observed equally in both sides. The reason could be photoexacerbation due to improper photoprotection. Markham et $\mathrm{al}^{15}$ had reported that a similar percentage of patients in each group (PUVA, 80\%; NBUVB, 75\%) developed grade 1 erythema showing that the regimens were equally erythemogenic; however, grade 2 erythema occurred only in PUVA group. Gordon et $\mathrm{al}^{6}$ observed an increased percentage of erythemal episodes with NB-UVB as compared to PUVA $(73 \%$ vs. $35 \%)$ while in contrast Yones et al 8 observed a significantly increased frequency of erythema with PUVA than NB-UVB (49\% vs. $22 \%$, $\mathrm{p}=.004)$.

Exacerbation of pruritus (PUVA 22.2\%, NB-UVB 18.5\%) and PMLE (PUVA 7.4\%, NBUVB 7.4\%) was observed equally with both modalities while nausea in $22.2 \%$ of patients was observed solely due to methoxypsoralen ingestion. This was comparable to observations made by Markham et al ${ }^{15}$ who also concluded that pruritus and polymorphic light eruption occurred equally in both groups, but only patients in the PUVA group developed nausea.

Three patients (10\%) were lost to followup which could be due to economic constraints, family and social obligations. This may be a handicap in widespread use of NB-UVB and PUVA as treatment modality.

\section{CONCLUSIONS}

It is therefore concluded from the present study that NBUVB and PUVA are equally effective in treatment of severe chronic plaque psoriasis in patients of Nepali and Indian origin.

Considering the good therapeutic efficacy, no drug related side effects, the low profile of acute and possibly also longterm side effects and the safe use in children and pregnancy, the present study considers narrowband UVB as first-line treatment for moderate-to-severe plaque psoriasis. PUVA on the other hand remains the mainstay of treatment for severe recalcitrant psoriasis.

The optimum treatment protocol for narrowband UVB and, in particular, the average duration of remission is yet to be determined in larger patient cohorts. In addition, prospective followup studies are required to assess the longterm risks in humans associated with therapeutic exposures to narrow band UVB radiation.

\section{REFERENCES}

1. Griffiths CEM, Camp RDR, Barker JNWN. Psoriasis. In: Rook's textbook of dermatology. Burns T, Brathnach S, Cox N. eds. $7^{\text {th }}$ edn. Oxford: Blackwell Science 2005:35(1):3569.

2. Tanew A, Rodakovic-Fijan S, Schemper M, et al. Narrow band UV-B phototherapy vs photochemotherapy in the treatment of chronic plaque-type psoriasis. Arch Dermatol 1999;135(5):519-24.

3. van Weelden $\mathrm{H}$, van der Leun JC. Improving the effectiveness of phototherapy for psoriasis. Br J Dermatol 1984;111:484-6. 
4. Dawe RS, Cameron H, Yule S, et al. A randomized controlled trial of narrowband ultraviolet $B$ vs bath-psoralen plus ultraviolet A photochemotherapy for psoriasis. $\mathrm{Br} \mathrm{J}$ Dermatol 2003;148(6):1194-204.

5. Snellman E, Klimenko T, Rantanen T. Randomized half-side comparison of narrowband UVB and trimethylpsoralen bath plus UVA treatments for psoriasis. Acta Derm Venereol 2004;84(2):132-7.

6. Gordon PM, Diffey BL, Matthews JN. A randomized comparison of narrow-band TL-01 phototherapy and PUVA photochemotherapy for psoriasis. J Am Acad Dermatol 1999;41(5 Pt 1):728-32.

7. Fredriksson T, Pettersson U. Severe psoriasis: oral therapy with a new retinoid. Dermatologica 1978;157(4):238-44.

8. Yones SS, Palmer RA, Garibaldinos TT, et al. Randomized double-blind trail of the treatment of chronic plaque psoriasis: efficacy of psoralen-UV-A therapy vs narrowband UV-B therapy. Arch Dermatol 2006;142(7):836-42.

9. Dogra S, Kanwar AJ. Narrow band UVB phototherapy in dermatology. Indian J Dermatol Venereol Leprol 2004;70(4):205-9.
10. Gupta AK, Anderson TF. Skin types and recommended total and incremental UVA exposure according to skin types. J Am Acad Dermatol 1987;17:706.

11. Lynch WS, Roenigk HH. Essentials of PUVA therapy. Guidelines for photochemotherapy. Cutis 1977;20(4):494501.

12. van Weelden H, De La Faille BH, Young E, et al. Comparison of narrow-band UV-B phototherapy and PUVA photochemotherapy in the treatment of psoriasis. Acta Derm Venereol 1990;70(3):212-5.

13. Cameron H, Dawe RS, Yule S, et al. A randomized, observer blinded trial of twice weekly narrowband ultraviolet $\mathrm{B}$ phototherapy for chronic plaque psoriasis. Br J Dermatol 2002;147(5):973-8.

14. Tahir R, Mujtaba G. Comparative efficacy of psoralen UVA photochemotherapy versus narrow band UVB phototherapy in the treatment of psoriasis. J Coll Physicians Surg Pak 2004;14(10):593-5.

15. Markham T, Rogers S, Collns P. Narrowband UV-B (TL-01) phototherapy vs oral 8-methoxypsoralen psoralen-UV-A for the treatment of chronic plaque psoriasis. Arch Dermatol 2003;139(3):325-8. 